\title{
TRPM2-AS promotes bladder cancer-genesis by targeting miR-22-3p thus releasing GINS2 mRNA
}

\section{Yudong Tian ( $\nabla$ tianyudong16@163.com )}

Zhengzhou University First Affiliated Hospital https://orcid.org/0000-0003-3978-1169

\section{Yanbin Guan}

Henan University of Traditional Chinese Medicine

\section{Yang Su}

Zhengzhou University First Affiliated Hospital

\section{Tao Yang}

Zhengzhou University First Affiliated Hospital

\section{Haizhou Yu}

Zhengzhou University First Affiliated Hospital

\section{Primary research}

Keywords: TRPM2-AS, miR-22-3p, GINS2, bladder cancer

Posted Date: April 29th, 2020

DOI: https://doi.org/10.21203/rs.3.rs-24770/v1

License: (c) (i) This work is licensed under a Creative Commons Attribution 4.0 International License. Read Full License 


\section{Abstract}

Background: We aimed to study the effects of IncRNA TRPM2-AS in bladder cancer (BLCA) by interacting with its downstream effectors miR-22-3p and GINS2 mRNA.

Methods: Online bioinformatic tools were used to identify the key IncRNA, miRNA and mRNA of interest in BLCA. TRPM2-AS, miR-22-3p and GINS2 mRNA expression was measured by qRT-PCR in bladder tissues and selected cell lines. Subcellular localization of TRPM2-AS in T24 and 5637 cell lines was identified using a cellular fractionation method. Luciferase reporter assay, RIP assay and RNA pull-down assay were employed to validate the direct binding relationship between TRPM2-AS, miR-22-3p and GINS2 mRNA. Cell viability, proliferation and apoptosis were measured by a series of cell functional experiments in T24 and 5637 cells.

Results: TRPM2-AS was primarily located in cell cytoplasm and significantly up-regulated in BLCA tissues and cell lines. TRPM2-AS knockdown significantly inhibited cell viability and proliferation, but promoted cell apoptosis. miR-22-3p, a significant downstream target of TRPM2-AS, showed a lower expression level in BLCA tissues and cell lines. miR-22-3p inhibition resulted in a significant enhancement of BLCA cancer cell phenotypes. Lastly, GINS2 mRNA was a downstream target of miR-22-3p, and was significantly upregulated in BLCA. The knockdown of GINS2 led to a significant suppression of BLCA cancer cell phenotypes.

Conclusions: TRPM2-AS was a tumor promoter and fulfilling its role through binding to miR-22-3p to increase GINS2 expression. This novel interactome in BLCA might become a new therapy in BLCA.

\section{Background}

Bladder cancer (BLCA) is a urinary system-originated malignancy, ranking 10 among all cancers all over the world [1], happening more frequently in males [1]. Although the occurrence and death rate of BLCA is usually the highest in South Europe, the rate of occurrence and death in China has been obviously increased within the recent 10 years [1, 2]. At present, surgery, chemotherapy and radiation therapy are employed to treat BLCA while the survival rate of BLCA patients have not been improved apparently $[3,4]$. Therefore, gene biomarkers and target therapy have been developed, and it is imperative to discover new targets to diagnose and treat BLCA.

Long non-coding RNA (IncRNA), containing more than 200 bp of nucleotides, has been demonstrated to be an essential participant during the development of malignancies [5-9]. The host gene of TRPM2-AS, transient receptor potential cation channel subfamily M member 2 antisense RNA, consists of 3 exons and locates at chromosome 21q22.3. TRPM2-AS was first discovered to be up-regulated in melanoma in 2008 [10], and it was gradually found that to be a cancer-promoting gene in more other tumors including reproductive system tumors, digestive system tumors and respiratory system tumors [11-17]. Yet, there have been no researches exploring how TRPM2-AS implements a marked effect in BLCA. Therefore, we are interested in studying the mechanism of TRPM2-AS in BLCA. 
In the last ten years researches have demonstrated that a plethora of small RNAs participate in the progression of human neoplasms [18]. Small RNAs include miRNAs, small interfering RNAs PIWI-related RNAs, and they are usually at the length of 18-29 nucleotides $[19,20]$. Among them, miRNAs have been frequently referred to important gene expression regulators and play essential roles in cancer progression. During the last three years, miR-22-3p's role in BLCA started to emerge and be studied, although it had been discovered to act as a tumor suppressor in neoplasms that occur in reproductive system, digestive system and respiratory system before [21-27]. But it is no research regarding the involvement of miR-22$3 p$ in a IncRNA-related interactome complex in BLCA. Thus, we aim to study a novel IncRNA-miR-22-3p interactome in BLCA.

The host gene of GINS2 (GINS complex subunit 2) locates on chromosome 16q24.1, and consists of five exons that belongs to GINS complex family [28]. GINS complex is a member of DNA replication helicase family that participates in the initiation of chromosome replication [29, 30]. At present, various studies have uncovered that GINS2 was anomalous to promote cancer progression [31-39]. However, the function of GINS2 in BLCA has not been studied.

We aimed to study the potential BLCA-regulatory mechanism of TRPM2-AS, miR-22-3p and GINS2 mRNA interactome. We hypothesized that TRPM2-AS was a tumor promoter through binding miR22-3p to increase GINS2 mRNA expression in BLCA. Our results may provide a novel diagnosis and therapy approach to BLCA.

\section{Material And Methods}

\section{Bioinformatic analysis}

GEPIA (http://gepia.cancer-pku.cn), an online gene expression profiling interactive analysis tool, was used to analyze the expression of TRPM2-AS and GINS2 mRNA in tumor and normal bladder tissues.

GSE37815 data series downloaded from GEO database provided the mRNA expression profile in bladder cancer. ENCORI (http://starbase.sysu.edu.cn/panCancer.php) was used to predict the miRNAs that could bind to TRPM2-AS, and TargetScan Human v7.2 algorithm was used to predict the target regulatory miRNAs that could bind to the 3'UTR of GINS2 mRNA.

\section{Tissue samples collection}

We collected BLCA tissues and corresponding adjacent tissues of 38 patients who were diagnosed with BLCA in The First Affiliated Hospital of Zhengzhou University hospital during the last three years. The processing of clinical tissue samples is in strict compliance with the ethical standards of the Declaration of Helsinki, and the ethics committee of the authors' institutes. All the patients signed the informed content, and their clinical characteristics were summarized in Table 1.

\section{qPCR}


Total miRNA was dissociated using miRcute miRNA Isolation Kit (DP501, Tiangen Biochemical, China) based on the manufacture protocol with checking the purity of the RNA by using gel electrophoresis. MiR22-3p reverse transcription was conducted using miRcute Plus miRNA First-Strand cDNA Kit (KR211, Tiangen Biochemical, China), while TRPM2-AS and GINS2 reverse transcription was carried out following the instruction of PrimeScript RT reagent Kit (Takara, RR037A, Japan). After reverse transcription, quantitative PCR was conducted using TB Green Premix Ex Taq II (Takara, RR820A, Japan). $2^{-\triangle \Delta C t}$ method was used to calculate RNA levels. We used GAPDH as the reference gene for TRPM2-AS and GINS2 mRNA, and U6 for miR-22-3p. All the primers were provided by GeneCopoeia (Guangzhou, China), and their sequences were provided in Table 2.

\section{Cell line acquisition and cell culture}

Both cancerous and normal cell lines were used in this study. The human BLCA cell lines RT4, T24, J82 and 5637, and normal urothelial epithelial cell line SV-HUC-1 were all acquired from the Cell Resource Center, Institute of Basic Medical Sciences, Chinese Academy of Medical Sciences. They were cultured in RPMI 1640 media with $10 \%$ serum in a humidified incubator with $5 \% \mathrm{CO}_{2}$ at $37^{\circ} \mathrm{C}$.

\section{Subcellular fractionation location}

Cytoplasmic and nuclear RNA of T24 and 5637 cells was separated by Invitrogen PARIS Kit (ThermoFisher, AM1921, USA) following specifications with GAPDH serving as a cytoplasmic control and U2 as a nuclear control. The expression levels of TRPM2-AS, GAPDH, and U2 were measured using qPCR. Each experimental procedure was processed at least three times.

\section{Cell transfection}

Negative control (NC) plasmids, miR-22-3p inhibitor, and small interfering RNAs for TRPM2-AS (siTRPM2-AS) and GINS2 (si-GINS2) were acquired from GeneCopoeia (Guangzhou, China). The transfection of these molecules into T24 and 5637 cells (plated in 6-well plates, $3 \times 10^{4} / \mathrm{mL}$ ) was conducted using Lipofectamine 2000 reagent (Cat\#: 11668027, ThermoFisher, USA). Then transfection lasted 48 hours.

\section{Cell viability assay}

Cell viability was detected using WST-8 method. Briefly, 4,800 transfected T24 and 5637 cells were seeded into 96 -well plates, and grown for $24,48,72$ hours, respectively. The $10 \mu \mathrm{L}$ cell counting kit-8 reagent (MedChemExpress, HY-K0301, China) was added to the cells for $4 \mathrm{~h}$ in the cell culture incubator. Lastly, the optical absorbance of every well was read at $450 \mathrm{~nm}$ under a microplate reader.

\section{BrdU incorporation ELISA assay}

BrdU DNA incorporation assay was used to evaluate cell proliferation ability. $3 \times 10^{4} / \mathrm{mL}$ T24 and 5637 cells were seeded into 96-well plates to grow for a day, and 10 $\mu \mathrm{L} \mathrm{BrdU} \mathrm{(5-bromo-2'-deoxyuridine,} \mathrm{a}$ 
thymidine analog, Abcam, ab126556, UK) was added to each well for $4 \mathrm{~h}$. Thereafter, the fixing solution was added to each well to denature the cellular DNA. Then, every well was aspirated and washed. 100 $\mu \mathrm{L} /$ well anti-BrdU primary antibody was added to every well to allow a $1 \mathrm{~h}$ reaction. Every well was aspirated and washed again, and $100 \mu \mathrm{L} /$ well Peroxidase conjugate Goat Anti-Mouse IgG secondary was added to incubate for $0.5 \mathrm{~h} .100 \mu \mathrm{L} /$ well TMB Peroxidase substrate was added and incubated for $0.5 \mathrm{~h}$ in the dark. The BrdU incorporated cells will be visible in blue, and the intensity of the blue color represents the proliferating abilities. To further quantify the proliferation, $100 \mu \mathrm{L}$ of Stop Solution was added to each well, and the optical absorbance of each well was read at $450 \mathrm{~nm}$ under a spectrophotometric microtiter plate reader. All procedures were conducted at room temperature, and experiments in every group were repeated for at least three times.

\section{Flow cytometry assay}

Flow cytometry was an important method to measure cell death and identify the early and late apoptotic cells. Briefly, $1 \times 10^{5} / \mathrm{mL}$ T24 and 5637 cells in every group were rinsed in PBS for three times, and fixed in cold methanol for $30 \mathrm{~min}$ at $4{ }^{\circ} \mathrm{C}$. Subsequently, the cells were suspended in $100 \mu \mathrm{l} 1 \times$ binding buffer. PBS was subsequently added to each well to wash the cell suspension for three times. Then, the cells were incubated with $5 \mu \mathrm{L}$ Annexin V-FITC and PI staining solution for $20 \mathrm{~min}$ in the dark. The cells then went through the FACS scan flow cytometer (Cytoflex, Beckman Coulter) within the next one hour. Both early (Annexin $\mathrm{V}$ positive and PI negative populations) and late apoptosis (Annexin V positive and PI positive staining) rate were counted as the apoptosis rate in this study.

\section{Caspase- 3 activation assay}

Caspase-3 activation is an essential process during cell apoptosis. This assay was conducted in our study to further investigate into the cell apoptosis conditions in every group. The caspase-3 activation was assessed using a Colorimetric Caspase-3 Assay Kit (Cat\#: ab39401, Abcam, UK). Briefly, 1×106 transfected T24 and 5637 cells were suspended in $50 \mu \mathrm{L}$ cold cell lysis buffer for 10 minutes. The protein concentration was detected and adjusted to $\sim 150 \mu$ g protein /50 $\mu \mathrm{L}$ reaction buffer. Then, $50 \mu \mathrm{L}$ reaction buffer containing $10 \mathrm{mM}$ DTT and $5 \mu \mathrm{L} 4 \mathrm{mM}$ DEVD-p-NA substrate was added to the cell lysates for an hour. Lastly, the optical absorbance was read at $405 \mathrm{~nm}$ using a spectrometer.

\section{Luciferase reporter assay}

The plasmids containing the wild strains and mutants of TRPM2-AS and GINS2 mRNA that contain the predicted binding sequences were constructed by and purchased from GeneCopoeia (China). These plasmids were transfected into the T24 and 5637 cells using Lipofectamine 2000 reagent (Cat\#: ThermoFisher, 11668027, USA). Then, miR-22-3p mimic or NC were co-transfected into the transfected T24 and 5637 cells using Lipofectamine 2000 reagent. $48 \mathrm{~h}$ later, the cells were gathered to be lysed using lysis buffer. The dual-luciferase reporter assay system (Cat\#: LF031, GeneCopoeia, China) was used to analyze the relative luciferase activity. 


\section{RNA immunoprecipitation (RIP)}

RIP immunoprecipitation assay was performed using the Magna RIP RNA-Binding Protein Immunoprecipitation Kit (Millipore, 17-700, USA). Briefly, the BLCA cells were lysed in RIP lysis buffer including magnetic beads conjugated with anti-Argonaute2 (AGO2) or anti-IgG antibodies. Samples were incubated with Proteinase $\mathrm{K}$ and the immunoprecipitated RNA was isolated with TRIzol Reagent (ThermoFisher, 15596026, USA). Finally, the enrichment of TRPM2-AS and miR-22-3p was measured by qRT-PCR.

\section{RNA pull-down assay}

The $5 \times 10^{6} \mathrm{~T} 24$ and 5637 cells were treated with $0.5 \mathrm{~mL}$ of $25 \mathrm{mM}$ Tris- $\mathrm{HCl}, 0.05 \% \mathrm{NP}-40,70 \mathrm{mM} \mathrm{KCl}, 2.5$ $\mathrm{mM}$ EDTA, $80 \mathrm{U} / \mathrm{mL}$ RNase inhibitor and $1 \times$ protease inhibitor cocktail on ice for 20 minutes. After centrifugation, the supernatant was collected and incubated with biotin-coupled miR-22-3p (bio-miR-22$3 p$ ) or biotin-coupled NC (bio-NC) for 2 hours. After incubation, the cell lysate containing bio-miR-22-3p or bio-NC was incubated with streptavidin magnetic beads. After 4 hours, the beads were washed with reaction buffer to isolate the bound RNAs in the pull-down complex, and the expression of pull-down RNA was detected by qRT-PCR.

\section{Western blot assay}

The protein of cells was extracted using RIPA Lysis Buffer containing 5 mM EDTA and PMSF (P0013B, Beyotime, China). Different groups of proteins were quantified by a spectrophotometer so that the proteins were at the same level. $20 \mu \mathrm{g}$ of total protein in these groups was separated by $10 \%$ SDS-PAGE gel. Total protein was transferred onto PVDF membranes (Millipore, MA, USA) with 5.0\% evaporated milk handled for 1 hour at $37^{\circ} \mathrm{C}$. Next, PVDF membranes were incubated with the primary antibodies against GINS2 (1:500, Cat\#: ab197123, Abcam, UK) and GAPDH (1:5000, Cat\#: ab175781, Abcam, UK) at $4^{\circ} \mathrm{C}$ overnight. Next day, the PVDF membranes were washed with PBS three times and incubated with Goat Anti-Rabbit IgG H\&L secondary antibody (1:5000, Cat\#: ab205718, Abcam, UK) at room temperature for 1 hour. ECL Substrate Kit (Cat\#: ab133406, Abcam, UK) was used to strengthen the band signals. FluorChem FC2 software (Media Cybernetics, Inc., Rockville, MD, USA) was used to read the intensities of the protein bands.

\section{Statistical analyses}

We analyzed our results using the Graphpad Prism v7.0 software. Data came from at least three independent results of every experiment, and were presented as mean $\pm S D$. The Wilcoxon test method was used to identify the significance of the differences between two groups, while one-way ANOVA with Dunnett's post hoc method was used to identify the significance of the differences among multiple groups. 0.05 was the cut-off value for statistical significance.

\section{Results}




\section{The identification of genes of interest in this study}

By interrogating GEPIA database, we found that IncRNA TRPM2-AS was significantly upregulated $(\log 2|\mathrm{FC}|=1.892, \mathrm{q}=1.64 \times 10-6)$ in bladder cancer (Figure 1A), implying its positive association with BLCA progression. Interestingly, TRPM2-AS has been proved to be a tumor promoter in colon cancer [13], gastric cancer [12], breast cancer [14] and lung cancer [17] by interacting with miRNAs; however, it has never been studied in BLCA. By interrogating GSE37815 data series from GEO database, we found that GINS2 was among the top 5 most DEGs with a $\log 2 \mathrm{FC}=1.99$ and adjusted $\mathrm{P}$ value $=0.00462$. Like TRPM2-AS, we found that GINS2 was considered to be a significant tumor promoter in thyroid cancer [32] lung cancer [33], cervical cancer [40], ovarian cancer [38], breast cancer [34], triple-negative breast cancer [35], and leukemia [39]. Based on these findings, we thus hypothesized that GINS2 might be a tumor promoter in BLCA as well. Figure 1C-D shows the relative expression of TRPM2-AS and GINS2 in BLCA tissues and the healthy tissues. Both TRPM2-AS and GINS2 were found to be significantly upregulated in BLCA tissues. Lastly, to identify a potential bridge miRNA between TRPM2-AS and GINS2, we intersected the predicted targets of TRPM2-AS from ENCORI starbase and those of GINS2 from TargetScan Human 7.2. Among the top 5 out of the total 13 common miRNAs, we found that miR-22-3p was once considered to suppress BLCA [41].

\section{TRPM2-AS silence suppressed cell viability and cell proliferation, but enhanced cell apoptosis in BLCA cells}

To explore the function of TRPM2-AS in BLCA, we first detected the expression of TRPM2-AS in BLCA tissues from 38 patients who were diagnosed with BLCA. The results showed that the expression of TRPM2-AS was increased by 3.6-fold in BLCA tissues compared with normal adjacent tissues (Figure 2A). Similar to BLCA tissues, we found that TRPM2-AS expression was higher in four BLCA cell lines (RT4, T24, J82 and 5637) than in normal urothelial epithelial cell line (SV-HUC-1) (Figure 2B). Due to the highest expression of TRPM2-AS in T24 and 5637 cell lines, the T24 and 5637 cell lines were chosen to conduct our study in depth. Then, the subcellular fractionation location assay was employed to observe TRPM2AS location in T24 and 5637 cell lines. The results showed that TRPM2-AS was majorly located in cytoplasm (Figure 2C). Then, si-TRPM2-AS and NC plasmids were used to transfect T24 and 5637 cells, showing that si-TRPM2-AS led to almost 70\% decrease of TRPM2-AS expression in T24 and 5637 cells compared with blank group (Figure 2D). In CCK-8 and BrdU assay, the cell viability and cell proliferation were suppressed in si-TRPM2-AS group compared with blank groups (Figure 2E-F). Then, we employed flow cytometry and caspase-3 assay to observe the effect of si-TRPM2-AS on cell apoptosis in two BLCA cell lines. The flow cytometry assay results demonstrated that the apoptosis rate in si-TRPM2-AS group was raised by approximately 2-fold in the two BLCA cell lines compared with blank groups (Figure 2G). The caspase-3 activity in si-TRPM2-AS group was raised by approximately 3 -fold in the two BLCA cell lines (Figure $2 \mathrm{H}$ ).

\section{MiR-22-3p is a downstream target of TRPM2-AS}


By interrogating ENCORI, the binding site between TRPM2-AS and miR-22-3p was discovered (Figure 3A). Luciferase reporter assay demonstrated that the fluorescence intensity was obviously reduced by half in co-transfection of TRPM2-AS-wild type and miR-22-3p mimics while other groups had no differences in T24 and 5637 cell lines (Figure 3B). Equally, RIP assay proved the TRPM2-AS expression with 4.6-fold and 5.1-fold increase in T24 and 5637 cells, indicating that TRPM2-AS could combine with miR-22-3p (Figure $3 \mathrm{C}$ ). In addition, we found that miR-22-3p expression was decreased by $65.7 \%$ in BLCA tissues compared with normal adjacent tissues (Figure 3D), at the same time, TRPM2-AS had a negative association with miR-22-3p (Figure 3E). By qRT-PCR, the miR-22-3p expression was decreased by more than $50 \%$ in T24 and 5637 cells compared with SV-HUC-1 cells (Figure 3F). And si-TRPM2-AS resulted in the miR-22-3p expression to increase by more than 1.5-fold in T24 and 5637 cells (Figure 3G).

\section{The effects of TRPM2-AS on BLCA cells by interacting with miR-22-3p}

To explore whether TRPM2-AS could regulate BLCA cells malignancy phenotypes by regulating miR-22$3 p$, we designed a series of rescue experiments. Before the experiments, the transfection efficiency of TRPM2-AS siRNA and miR-22p-3p inhibitor was confirmed using qPCR (Figure 4A). Firstly, CCK-8 assay results displayed that miR-22-3p inhibitor enhanced cell viability while si-TRPM2-AS weakened it. When the BLCA cells were co-transfected with miR-22-3p inhibitor and si-TRPM2-AS, the cell viability had no significant differences from the blank group (Figure 4B). Equally, BrdU incorporation assay showed that the cell proliferation ability in miR-22-3p inhibitor group increased by nearly 1.5 times, while the cell proliferation ability in co-transfection group was similar to blank group (Figure 4C). As for cell apoptosis, flow cytometry results revealed that the cell apoptosis rate was declined by approximately $60 \%$ in miR-22$3 p$ inhibitor group, while the cell apoptosis rate was raised by approximately 2 -fold in si-TRPM2-AS group in T24 and 5637 cell lines. The cell apoptosis rate in co-transfection group was the same as that in blank group (Figure 4D). We also employed caspase-3 assay to further study the effects of loss-of-function of TRPM2-AS and miR-22-3p. The caspase-3 assay results showed that miR-22-3p inhibitor led to $40 \%$ decrease of the caspase-3 activity, and si-TRPM2-AS led to 3-fold increase of the caspase-3 activity in T24 and 5637 cells. When miR-22-3p inhibitor and si-TRPM2-AS were co-transfected into the T24 and 5637 cells, there was no significant difference between the co-transfection group and blank group (Figure $4 \mathrm{E})$.

\section{GINS2 was a downstream target gene of miR-22-3p}

By interrogating TargetScan Human 7.2, the complementary sequences of GINS2 mRNA 3'UTR and miR22-3p was obtained (Figure 5A). Thereafter, we conducted dual luciferase reporter gene assay and RNA pull-down assay to verify the complementary binding relationship. The fluorescence intensity was obviously reduced in of GINS2-wild type and miR-22-3p mimics co-transfection group, while other groups had no differences in T24 and 5637 cell lines (Figure 5B). RNA pull-down assay also proved that miR-22$3 p$ could directly interact with GINS2 mRNA (Figure $5 C$ ). Interestingly, we found that GINS2 was significantly up-regulated in BLCA tissues compared with normal adjacent tissues (Figure 5D), indicating a a negative correlation with miR-22-3p expression (Figure 5E). In addition, the expression of GINS2 
mRNA increased by 6.2-fold in T24 cells and 5.7-fold in 5637 cells compared to SV-HUC-1 cells (Figure 5F). At protein level, GINS2 expression was raised by 1.9-fold in T24 cells and 1.7-fold in 5637 cells compared to SV-HUC-1 (Figure 5G). What's more, the transfection of miR-22-3p mimic resulted in 50\% decrease of the GINS2 expression, while the transfection of miR-22-3p inhibitor resulted in almost 2-fold increase of the GINS2 expression in T24 and 5637 cells (Figure $5 \mathrm{H}$ ).

\section{The suppression effects of GINS2 silence on BLCA cells malignancy phenotypes were reversed by miR- 22-3p inhibition}

To explore whether the effects of GINS2 on BLCA cells was regulated by miR-22-3p, the miR-22-3p inhibitor and si-GINS2 were transfected into T24 and 5637 cells. The transfection efficiency of miR-22-3p and GINS2 siRNA was confirmed using qPCR (Figure 6A) and western blot method. The results of western blot showed that the miR-22-3p inhibitor led to the approximately 1.5-fold increase of the expression of GINS2 protein, while the expression of GINS2 protein in the si-GINS2 group was suppressed by approximately $50 \%$, indicating that the miR-22-3p inhibitor and si-GINS2 were successfully transfected into both cell lines (Figure 6B). Then, the CCK-8 assay results showed that the si-GINS2 weakened cell viability in T24 and 5637 cells, but the co-transfection with miR-22-3p inhibitor attenuated the effect of siGINS2 on cell viability in the two cell lines (Figure 6C). Equally, BrdU incorporation assay results showed that the proliferation ability of T24 and 5637 cells was impaired by $50 \%$ in si-GINS2 group, but the proliferation ability of the two BLCA cell lines in co-transfection group was the same as the blank group (Figure 6D). For cell apoptosis, the flow cytometry analysis results revealed that the apoptosis rate induced by si-GINS2 was raised by 2.3-fold in T24 cells and 1.7-fold in 5637 cells compared to blank group, while the apoptosis rate in co-transfection group did not show any significant differences with blank group (Figure 6E). Furthermore, we observed the caspase-3 activity in the transfected BLCA cells, finding that the caspase-3 activation was enhanced by si-GINS2, and the caspase-3 activity in cotransfection group did not show a significant difference from the blank group either (Figure 6F).

\section{Discussion}

TRPM2-AS was significantly up-regulated in BLCA tissues and cells compared with normal control and majorly located in cell cytoplasm. We found TRPM2-AS enhanced the cell viability and cell proliferation, and impaired cell apoptosis in BLCA cells. Furthermore, TRPM2-AS had a negative association with miR22-3p, which had lower expression in BLCA tissues and cells to act as a negative regulator of BLCA progression. GINS2, validated to be a target gene of miR-22-3p, was found up-regulated in BLCA tissues and cells, and it also could promote cell viability and cell proliferation, while inhibited cell apoptosis.

Recently, IncRNA TRPM2-AS has been found to be aberrantly up-regulated in a wide spectrum of neoplasms. For instance, in melanoma, TRPM2-AS were over-expressed [10]. Its aberrant up-regulation led to significant cancer cell phenotypes, and its loss-of-function resulted in reduced cancer phenotypes. TRPM2-AS was found increased in prostate cancer, and the knockout of TRPM2-AS induced cell apoptosis with strengthening cell stress as well as cell cycle arrest [11]. Besides, TRPM2-AS was 
increased in gastric cancer, and had a significant association with invasion depth, lymphatic metastasis, TNM stage and 5-year survival rate. Furthermore, TRPM2-AS enhanced cell propagation, migration and invasion through inhibiting miR-195 to promote the development of gastric cancer [12]. Similarly, TRPM2AS with higher expression in colorectal cancer cells strengthened colorectal cancer cell proliferation via immediately combining with RNA-binding protein TAF15 thereby stabilizing the TRPM2 mRNA [13]. In addition, TRPM2-AS was reported to be up-regulated in breast cancer to promote uncontrollable proliferation and weaken breast cancer cells' apoptosis through binding with miR-140-3p [14]. Besides, TRPM2-AS was up-regulated in hepatocellular carcinoma (HCC) tissues than in normal adjacent tissues, and it had a significant correlation with tumor size, differentiation, and the prognosis outcome of HCC patients [16]. Compared with previous studies, our results had the similar conclusion that TRPM2-AS was up-regulated in BLCA to promote the cell viability and cell proliferation, and inhibit cell apoptosis. Our study not only identified the significant up-regulation of TRPM2-AS in bladder cancer, but also confirmed its promoting effects on BLCA phenotypes. Clearly, compared with previous studies, we need to collect more clinical data to verify the regulatory association between TRPM2-AS expression and the clinical characteristics.

miR-22-3p has been studied in a plethora of human neoplasms including BLCA, and it has been frequently considered as a significant tumor suppressor. For instance, miR-22-3p was reported to be significantly downregulated in $\mathrm{NCl}-\mathrm{H} 446$ (a small cell lung cancer cell line), and enhanced cell apoptosis while inhibited cell migration through down-regulating WRNIP1 [26]. Similarly, miR-22-3p was downregulated in HCC, leading to the malignant progression of HCC [27]. Interestingly, miR-22-3p was also found to hinder the malignancy phenotypes of BLCA [41]. Equal to these researches, we found that miR22-3p was significantly down-regulated in BLCA. At the same time, miR-22-3p could inhibit cell viability and cell proliferation while enhance cell apoptosis. We used a more accurate proliferation assay, BrdU incorporation assay, to study its effect on BLCA cell proliferation. Plus, we also detected the caspase-3 activation in BLCA cells to further reveal the effects of miR-22-3p on cell apoptosis. Our study confirmed that caspase-3 activation was involved in miR-22-3p-induced apoptosis process. This finding is similar to the study of Qinghai Zeng et al. [42], in which the cleaved caspase 3 protein level was reported to be upregulated in T24 and RT4 cell lines after the treatment of miR-22-3p mimic.

Besides, miR-22-3p has also been reported to participate in a more complex network, the competitive endogenous RNA (ceRNA) network. For instance, miR-22-3p was reported to be involved in a ceRNA network pathway that included IncRNA NNT-AS1 and YAP1, thus regulating the malignancy phenotypes of NSCLC cells [43]. In gastric cancer, miR-22-3p was reported to be regulated by IncRNA CTC-497E21.4 thus regulating NET1 expression to suppress the malignant development of gastric cancer [44]. Moreover, LINC00858 acted through ceRNA network to suppress miR-22-3p, thereby regulating the down-stream effector, YWHAZ, to strengthen the progression of colorectal cancer [25]. We herein validated the regulatory relationship between miR-22-3p and TRPM2-AS, and the latter promoted BLCA by suppressing the former. The ceRNA network in BLCA involving miR-22-3p has not yet been reported before, the present study presented a novel ceRNA network that involves miR-22-3p, which might open a new approach for target therapy of BLCA. 
GINS2 has been preliminarily studied in various human cancers such as that GINS2 enhanced cell proliferation and weakened cell apoptosis in thyroid cancer by regulating CITED2 and LOXL2 expression [32]. GINS2 was upregulated in NSCLC tissues and cell lines, indicating with the poor prognosis outcome of the patients. Moreover, GINS2 enhanced NSCLC cell propagation, migration, invasion and EMT by regulating the phosphorylation of PI3K p85, AKT, MEK and ERK [33]. Besides, GINS2 was over-expressed in triple negative breast cancer (TNBC) cell lines, and strengthened the aggression of TNBC cells through increasing MMP9 level [35]. What's more, GINS2 expression level was obviously higher in cervical cancer tissues and cell lines, indicating the poor survival outcome of patients, and promoted cell propagation, migration and invasion [37]. In epithelial ovarian cancer, GINS2 with highly expression in tissues enhanced cell propagation while mitigated cell cycle arrest in S phase [38]. To our knowledge, GINS2 has not yet been studied in BLCA before. We herein identified that GINS2 was a validated downstream effector of miR-22-3p. We also discovered a similar result to previous studies that GINS2 was an oncogene to promote the development of BLCA cells. We found that the effects of GINS2 knockdown on BLCA cell phenotypes could be attenuated by miR-22-3p inhibition.

In our study, we designed cell experiments to verify our hypothesis that a novel ceRNA network pathway that involves TRPM2-AS/miR-22-3p/GINS2 could regulate the viability, proliferation and apoptosis of BLCA cell phenotypes; however, we did not conduct experiments to study the effects of the ceRNA network pathway on BLCA cell aggression phenotypes such as migration and invasion. Besides, we didn't conduct animal models to further confirm our in vitro experiment results. In addition, the occurrence and progression of tumor involves many factors, we also need to discover other factors that interact with the novel interactome to expand the ceRNA network.

\section{Conclusion}

Our study suggested that TRPM2-AS was a tumor promoter, and enhanced the progression of bladder cancer by down-regulating miR-22-3p thus increasing GINS2 expression. TRPM2-AS/miR-22-3p/GINS2 might become a new therapy in bladder cancer in the future.

\section{Abbreviations}

miRNAs: microRNAs; PBS: phosphate bufered solution; EdU: 5-ethynyl-2'-deoxyuridine; BLCA: bladder cancer; qPCR: quantitative real-time PCR.

\section{Declarations}

\section{Ethics approval and consent to participate}

Ethic Committee of The First Affiliated Hospital of Zhengzhou University (Henan, China) approved the study.

\section{Consent for publication}


Informed consent was obtained from all patients.

\section{Availability of Data and Materials}

The datasets used during the current study are available from the corresponding author on reasonable request.

\section{Funding}

This research has received no funds.

Competing interests

There is no conflict of interest existed among the authors.

\section{Authors' contributions}

YDT designed the experiments. YBG and YS conducted the experiments. TY and HZY collected the data and wrote the manuscript.

\section{Acknowledgements}

Not applicable.

\section{References}

1. Bray F, Ferlay J, Soerjomataram I, Siegel RL, Torre LA, Jemal A: Global cancer statistics 2018: GLOBOCAN estimates of incidence and mortality worldwide for 36 cancers in 185 countries. $C A$ Cancer J Clin 2018, 68(6):394-424.

2. Chen W, Zheng R, Baade PD, Zhang S, Zeng H, Bray F, Jemal A, Yu XQ, He J: Cancer statistics in China, 2015. CA Cancer J Clin 2016, 66(2):115-132.

3. Humphrey PA, Moch H, Cubilla AL, Ulbright TM, Reuter VE: The 2016 WHO Classification of Tumours of the Urinary System and Male Genital Organs-Part B: Prostate and Bladder Tumours. Eur Urol 2016, 70(1):106-119.

4. Babjuk M: Trends in Bladder Cancer Incidence and Mortality: Success or Disappointment? Eur Urol 2017, 71(1):109-110.

5. Shi X, Sun M, Liu H, Yao Y, Song Y: Long non-coding RNAs: a new frontier in the study of human diseases. Cancer Lett 2013, 339(2):159-166.

6. Trimarchi T, Bilal E, Ntziachristos P, Fabbri G, Dalla-Favera R, Tsirigos A, Aifantis I: Genome-wide mapping and characterization of Notch-regulated long noncoding RNAs in acute leukemia. Cel/ 2014, 158(3):593-606. 
7. Cheng W, Zhang Z, Wang J: Long noncoding RNAs: new players in prostate cancer. Cancer Lett 2013, 339(1):8-14.

8. Zhong X, Lu M, Wan J, Zhou T, Qin B: Long noncoding RNA kcna3 inhibits the progression of colorectal carcinoma through down-regulating YAP1 expression. Biomed Pharmacother 2018, 107:382-389.

9. Wu X, Zhang P, Zhu H, Li S, Chen X, Shi L: Long noncoding RNA FEZF1-AS1 indicates a poor prognosis of gastric cancer and promotes tumorigenesis via activation of Wnt signaling pathway. Biomed Pharmacother 2017, 96:1103-1108.

10. Orfanelli U, Wenke AK, Doglioni C, Russo V, Bosserhoff AK, Lavorgna G: Identification of novel sense and antisense transcription at the TRPM2 locus in cancer. Cell Res 2008, 18(11):1128-1140.

11. Orfanelli U, Jachetti E, Chiacchiera F, Grioni M, Brambilla P, Briganti A, Freschi M, Martinelli-Boneschi $\mathrm{F}$, Doglioni $\mathrm{C}$, Montorsi $\mathrm{F}$ et al: Antisense transcription at the TRPM2 locus as a novel prognostic marker and therapeutic target in prostate cancer. Oncogene 2015, 34(16):2094-2102.

12. Huang B, Chang C, Wang BL, Li H: ELK1-induced upregulation of IncRNA TRPM2-AS promotes tumor progression in gastric cancer by regulating miR-195/ HMGA1 axis. J Cell Biochem 2019, 120(10):16921-16933.

13. Pan L, Li Y, Jin L, Li J, Xu A: TRPM2-AS promotes cancer cell proliferation through control of TAF15. Int J Biochem Cell Bio/ 2020, 120:105683.

14. Sun T, Song Y, Yu H, Luo X: Identification of IncRNA TRPM2-AS/miR-140-3p/PYCR1 axis's proliferates and anti-apoptotic effect on breast cancer using co-expression network analysis. Cancer Biol Ther 2019, 20(6):760-773.

15. Ma LY, Xie XW, Ma L, Pang JL, Xiong XM, Zheng HD, Shen XL, Wen ZG, Wang HY: Downregulated long non-coding RNA TRPM2-AS inhibits cisplatin resistance of non-small cell lung cancer cells via activation of p53-p66shc pathway. Eur Rev Med Pharmacol Sci 2017, 21(11):2626-2634.

16. Xu C, Huang Q, Zhang C, Xu W, Xu G, Zhao X, Liu X, Du Y: Long non-coding RNA TRPM2-AS as a potential biomarker for hepatocellular carcinoma. Ir J Med Sci 2018, 187(3):621-628.

17. Huang C, Qin Y, Liu H, Liang N, Chen Y, Ma D, Han Z, Xu X, Zhou X, He J et al: Downregulation of a novel long noncoding RNA TRPM2-AS promotes apoptosis in non-small cell lung cancer. Tumour Biol 2017, 39(2):1010428317691191.

18. Mattick JS: Non-coding RNAs: the architects of eukaryotic complexity. EMBO Rep 2001, 2(11):986991.

19. Ahadi A, Brennan S, Kennedy PJ, Hutvagner G, Tran N: Long non-coding RNAs harboring miRNA seed regions are enriched in prostate cancer exosomes. Sci Rep 2016, 6:24922.

20. Seok H, Ham J, Jang ES, Chi SW: MicroRNA Target Recognition: Insights from Transcriptome-Wide Non-Canonical Interactions. Mol Cells 2016, 39(5):375-381.

21. Zhang W, Zhan F, Li D, Wang T, Huang H: RGMB-AS1/miR-22-3p/NFIB axis contributes to the progression of gastric cancer. Neoplasma 2020. 
22. Li S, Chen Y, Xie L, Meng Y, Zhu L, Chu H, Gu D, Zhang Z, Du M, Wang M: Sex hormones and genetic variants in hormone metabolic pathways associated with the risk of colorectal cancer. Environ Int 2020, 137:105543.

23. Liang L, Xu J, Wang M, Xu G, Zhang N, Wang G, Zhao Y: LncRNA HCP5 promotes follicular thyroid carcinoma progression via miRNAs sponge. Cell Death Dis 2018, 9(3):372.

24. Qiu BQ, Zhang PF, Xiong D, Xu JJ, Long X, Zhu SQ, Ye XD, Wu Y, Pei X, Zhang XM et al: CircRNA fibroblast growth factor receptor 3 promotes tumor progression in non-small cell lung cancer by regulating Galectin-1-AKT/ERK1/2 signaling. J Cell Physiol 2019, 234(7):11256-11264.

25. Sha QK, Chen L, Xi JZ, Song H: Long non-coding RNA LINC00858 promotes cells proliferation, migration and invasion by acting as a ceRNA of miR-22-3p in colorectal cancer. Artif Cells Nanomed Biotechnol 2019, 47(1):1057-1066.

26. Jiang W, Han X, Wang J, Wang L, Xu Z, Wei Q, Zhang W, Wang H: miR-22 enhances the radiosensitivity of small-cell lung cancer by targeting the WRNIP1. J Cell Biochem 2019, 120(10):17650-17661.

27. Chen J, Wu FX, Luo HL, Liu JJ, Luo T, Bai T, Li LQ, Fan XH: Berberine upregulates miR-22-3p to suppress hepatocellular carcinoma cell proliferation by targeting Sp1. Am J Trans/ Res 2016, 8(11):4932-4941.

28. MacNeill SA: Structure and function of the GINS complex, a key component of the eukaryotic replisome. Biochem J 2010, 425(3):489-500.

29. Labib K, Gambus A: A key role for the GINS complex at DNA replication forks. Trends Cell Biol 2007, 17(6):271-278.

30. Kamada K: The GINS complex: structure and function. Subcell Biochem 2012, 62:135-156.

31. Huang J, Li Y, Lu Z, Che Y, Sun S, Mao S, Lei Y, Zang R, Li N, Zheng S et al: Analysis of functional hub genes identifies CDC45 as an oncogene in non-small cell lung cancer - a short report. Cell Oncol (Dordr) 2019, 42(4):571-578.

32. Ye Y, Song YN, He SF, Zhuang JH, Wang GY, Xia W: GINS2 promotes cell proliferation and inhibits cell apoptosis in thyroid cancer by regulating CITED2 and LOXL2. Cancer Gene Ther 2019, 26(3-4):103113.

33. Liu X, Sun L, Zhang S, Zhang S, Li W: GINS2 facilitates epithelial-to-mesenchymal transition in nonsmall-cell lung cancer through modulating PI3K/Akt and MEK/ERK signaling. J Cell Physio/2019.

34. Rantala JK, Edgren H, Lehtinen L, Wolf M, Kleivi K, Vollan HK, Aaltola AR, Laasola P, Kilpinen S, Saviranta $P$ et al: Integrative functional genomics analysis of sustained polyploidy phenotypes in breast cancer cells identifies an oncogenic profile for GINS2. Neoplasia 2010, 12(11):877-888.

35. Peng L, Song Z, Chen D, Linghu R, Wang Y, Zhang X, Kou X, Yang J, Jiao S: GINS2 regulates matrix metallopeptidase 9 expression and cancer stem cell property in human triple negative Breast cancer. Biomed Pharmacother 2016, 84:1568-1574.

36. Zheng M, Zhou Y, Yang X, Tang J, Wei D, Zhang Y, Jiang JL, Chen ZN, Zhu P: High GINS2 transcript level predicts poor prognosis and correlates with high histological grade and endocrine therapy 
resistance through mammary cancer stem cells in breast cancer patients. Breast Cancer Res Treat 2014, 148(2):423-436.

37. Ouyang F, Liu J, Xia M, Lin C, Wu X, Ye L, Song L, Li J, Wang J, Guo P et al: GINS2 is a novel prognostic biomarker and promotes tumor progression in early-stage cervical cancer. Oncol Rep 2017, 37(5):2652-2662.

38. Yan T, Liang W, Jiang E, Ye A, Wu Q, Xi M: GINS2 regulates cell proliferation and apoptosis in human epithelial ovarian cancer. Oncol Lett 2018, 16(2):2591-2598.

39. Zhang X, Zhong L, Liu BZ, Gao YJ, Gao YM, Hu XX: Effect of GINS2 on proliferation and apoptosis in leukemic cell line. Int J Med Sci 2013, 10(12):1795-1804.

40. Kacich RL, Williams LT, Coughlin SR: Arachidonic acid and cyclic adenosine monophosphate stimulation of $\mathrm{c}$-fos expression by a pathway independent of phorbol ester-sensitive protein kinase $\mathbf{C}$. Mol Endocrinol 1988, 2(1):73-77.

41. Xu M, Li J, Wang X, Meng S, Shen J, Wang S, Xu X, Xie B, Liu B, Xie L: MiR-22 suppresses epithelialmesenchymal transition in bladder cancer by inhibiting Snail and MAPK1/Slug/vimentin feedback loop. Cell Death Dis 2018, 9(2):209.

42. Zeng Q, Liu J, Cao P, Li J, Liu X, Fan X, Liu L, Cheng Y, Xiong W, Li J et al: Inhibition of REDD1 Sensitizes Bladder Urothelial Carcinoma to Paclitaxel by Inhibiting Autophagy. Clin Cancer Res 2018, 24(2):445-459.

43. He W, Zhang Y, Xia S: LncRNA NNT-AS1 promotes non-small cell lung cancer progression through regulating miR-22-3p/YAP1 axis. Thorac Cancer 2020.

44. Zong W, Feng W, Jiang Y, Cao Y, Ke Y, Shi X, Ju S, Cong H, Wang X, Cui M et al: LncRNA CTC497E21.4 promotes the progression of gastric cancer via modulating miR-22/NET1 axis through RhoA signaling pathway. Gastric Cancer 2020, 23(2):228-240.

\section{Tables}

Table 1. The clinical characteristics of 38 patients with bladder cancer. 


\begin{tabular}{|c|c|c|}
\hline characteristics & Total = 32 & Percentage (\%) \\
\hline \multicolumn{3}{|l|}{ Age(years) } \\
\hline$\leq 60$ & 20 & $52.6 \%$ \\
\hline$>60$ & 18 & $47.4 \%$ \\
\hline \multicolumn{3}{|l|}{ Gender } \\
\hline Male & 23 & $60.5 \%$ \\
\hline Female & 15 & $39.5 \%$ \\
\hline \multicolumn{3}{|c|}{ Tumor diameter(cm) } \\
\hline$\leq 3$ & 21 & $55.3 \%$ \\
\hline$>3$ & 17 & $44.7 \%$ \\
\hline \multicolumn{3}{|c|}{ Histological grade } \\
\hline Low & 20 & $52.6 \%$ \\
\hline High & 18 & $47.4 \%$ \\
\hline \multicolumn{3}{|l|}{ TNM stage } \\
\hline I & 8 & $21.1 \%$ \\
\hline II & 17 & $44.7 \%$ \\
\hline III & 9 & $23.7 \%$ \\
\hline IV & 4 & $10.5 \%$ \\
\hline \multicolumn{3}{|c|}{ Lymphatic metastasis } \\
\hline Positive & 16 & $42.1 \%$ \\
\hline Negative & 22 & $57.9 \%$ \\
\hline
\end{tabular}

\section{Table 2. The primer sequences for RT-qPCR}




\begin{tabular}{ll} 
GENE & Primer sequences $\left(5^{\prime}\right.$-3 $\left.^{\prime}\right)$ \\
\hline TRPM2-AS & Forward: AAATGGCCATGCAGGTCGAA \\
\cline { 2 - 2 } & Reverse: TATTTGCCCTCGTAACCGCA \\
\hline miR-22-3p & Forward: CGAAGCTGCCAGTTGAAGAA \\
\hline \multirow{2}{*}{ U6 } & Reverse: TGGTGTCGTGGAGTCG \\
\hline \multirow{2}{*}{ GINS2 } & Forward: CTCGCTTCGGCAGCACA \\
\cline { 2 - 2 } & Reverse: AACGCTTCACGAATTTGCGT \\
\hline GAPDH & Feverse: CACATGCGCATTCTCAGAGC \\
\hline & Reverse: GGCTGTTGTCATACTTCTCATGG
\end{tabular}

Figures 
A GEPIA data

\begin{tabular}{|c|c|c|c|c|c|}
\hline Gene Symbol & Gene ID & Median (tumor) & Median (normal) & $\log _{2} \mathrm{FC}$ & adj.P \\
\hline ARAP1-AS1 & ENSG00000256007.1 & 18.904 & 0.687 & 3.561 & $6.99 \mathrm{E}-13$ \\
\hline LINC01296 & ENSG00000244306.9 & 8.405 & 0.39 & 2.758 & $8.75 \mathrm{E}-13$ \\
\hline LINC00958 & ENSG00000251381.6 & 4.36 & 0.08 & 2.311 & 4.80E-04 \\
\hline KCNMB2-AS1 & ENSG00000237978.5 & 5.32 & 0.468 & 2.106 & $6.86 \mathrm{E}-10$ \\
\hline TRPM2-AS & ENSG00000230061.2 & 3.249 & 0.145 & 1.892 & $1.64 \mathrm{E}-06$ \\
\hline
\end{tabular}

B GSE37815 differentially expressed genes ranked by adj. P. Val

\begin{tabular}{|c|c|c|c|c|c|}
\hline ID & adj.P.Val & P.Value & $\log _{2} F C$ & Gene.symbol & Gene.title \\
\hline ILMN_1795470 & 0.000666 & 0.000000972 & 1.76 & MTFP1 & mitochondrial fission process 1 \\
\hline ILMN_1809590 & 0.00462 & 0.0000233 & 1.99 & GINS2 & GINS complex subunit 2 \\
\hline ILMN_1657347 & 0.00561 & 0.0000329 & 1.84 & PODXL2 & podocalyxin like 2 \\
\hline ILMN_1663390 & 0.00573 & 0.0000342 & 3.15 & CDC20 & cell division cycle 20 \\
\hline ILMN_1801661 & 0.00656 & 0.0000441 & 2.63 & KRT7 & keratin 7 \\
\hline
\end{tabular}
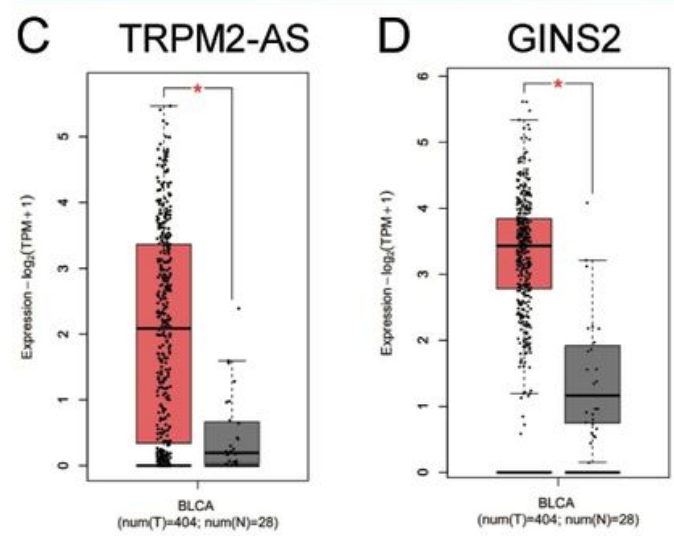

$\mathrm{E}$

ENCORI starbase predicted targets of TRPM2-AS
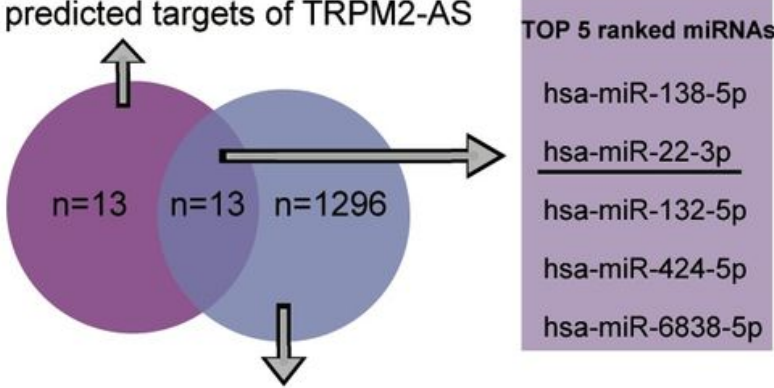

TargetScan Human 7.2 predicted targets of GINS2

\section{Figure 1}

The identification of interested interactome in BLCA. A. The top 5 most significantly upregulated IncRNAs in BLCA using GEPIA database based on the log2|FC| values. FC: fold change; adj.P: adjusted P value. B. The top five most significantly upregulated mRNAs in BLCA by analyzing GSE37815 data series. C. The relative expression of TRPM2-AS in BLCA tissues and healthy tissues from GEPIA database. D. The relative expression of GINS2 in BLCA tissues and healthy tissues from GEPIA database. E. The identification of the bridge miRNAs between TRPM2-AS and GINS2 mRNA. 
A
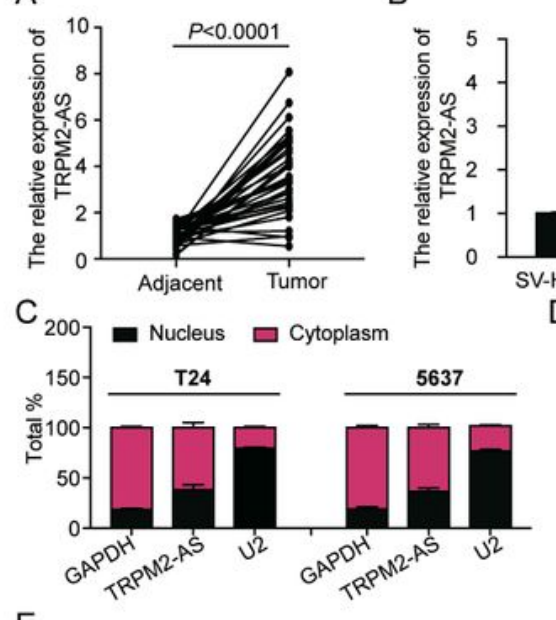

$\mathrm{E}$

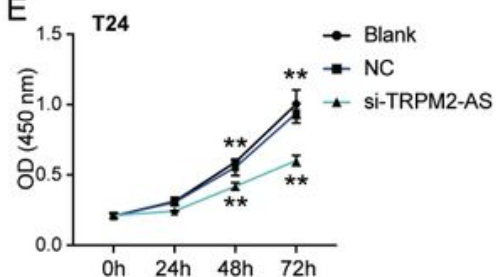

$\mathrm{F}$

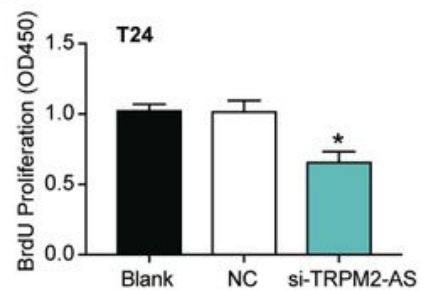

G

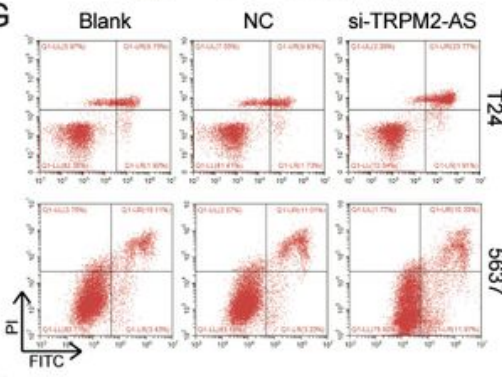

$\mathrm{H}$

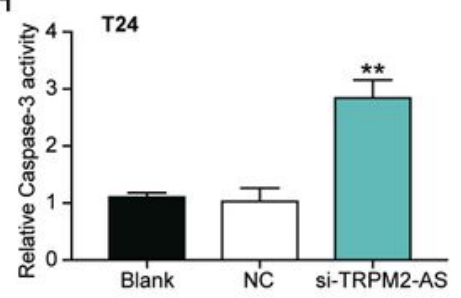

B

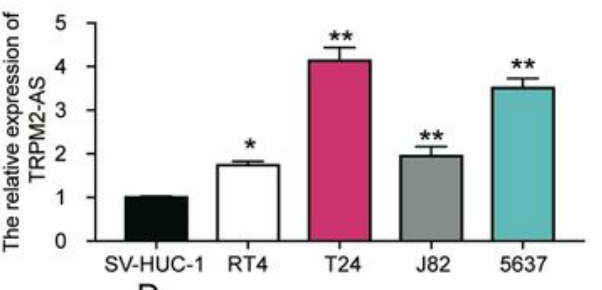

$D_{\text {¿ }} 1.57$ Blank $\square$ NC $\square$ si-TRPM2-AS
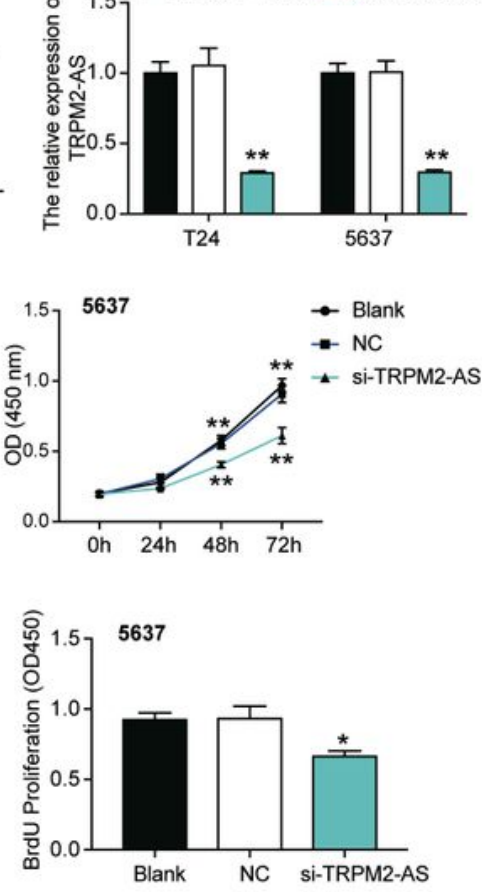

m Blank $\square$ NC $\square$ si-TRPM2-AS
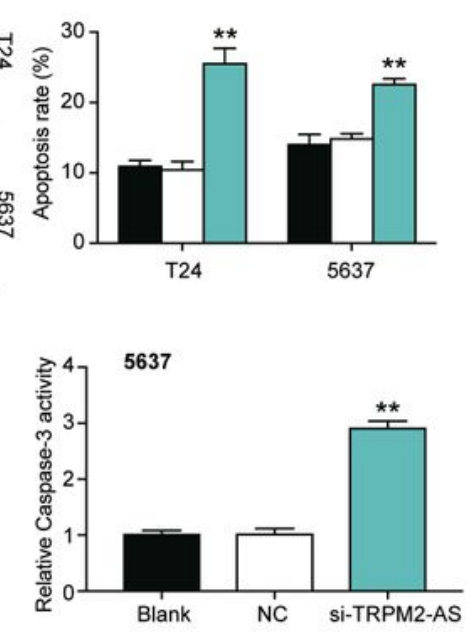

\section{Figure 2}

The inhibitory effect of si-TRPM2-AS on bladder cancer was confirmed. A. The expression of TRPM2-AS was increased in BLCA tissues compared with normal adjacent tissues. $N=38$, Student's t-test. $B$. The expression of TRPM2-AS was higher in four BLCA cell lines (RT4, T24, J82 and 5637) than that in normal urothelial epithelial cell line SV-HUC-1. ${ }^{*} P<0.05$, ${ }^{\star} \mathrm{P}<0.001$ compared with SV-HUC-1 cell, ANOVA. C. The location of TRPM2-AS was majorly in cytoplasm of T24 and 5637 cells. D. The transfection effectiveness 
in T24 and 5637 cell lines after transfecting si-TRPM2-AS, negative control and blank control. ${ }^{*} \mathrm{P}<0.001$ compared with blank group, ANOVA. E. CCK-8 assay was used to observe the cell viability in T24 and 5637 cell lines after transfecting si-TRPM2-AS, negative control and blank control at $0,24,48$, and 72 hours. ${ }^{*} \mathrm{P}<0.001$ compared with blank group, ANOVA. F. BrdU assay was used to observe the cell proliferation in T24 and 5637 cell lines after transfecting si-TRPM2-AS, negative control and blank control at 48 hours. ${ }^{*} \mathrm{P}<0.05$ compared with blank group, ANOVA. G. Flow cytometry was employed to measure the cell apoptosis after transfecting si-TRPM2-AS, negative control and blank control. ${ }^{* * P}<0.001$ compared with blank group, ANOVA. H. The caspase-3 activity assay was employed to measure the caspase-3 activity after transfecting si-TRPM2-AS, negative control and blank control. ** $\mathrm{P}<0.001$ compared with blank group, ANOVA. 


\section{A}

Wild-type TRPM2-AS 5' accgccacggccuUGGCAGCUl 3'

3' ugucaagaaguugACCGUCGAa 5'

Mutant TRPM2-AS

5' accgccacggccuACAUGAGAu 3'

B
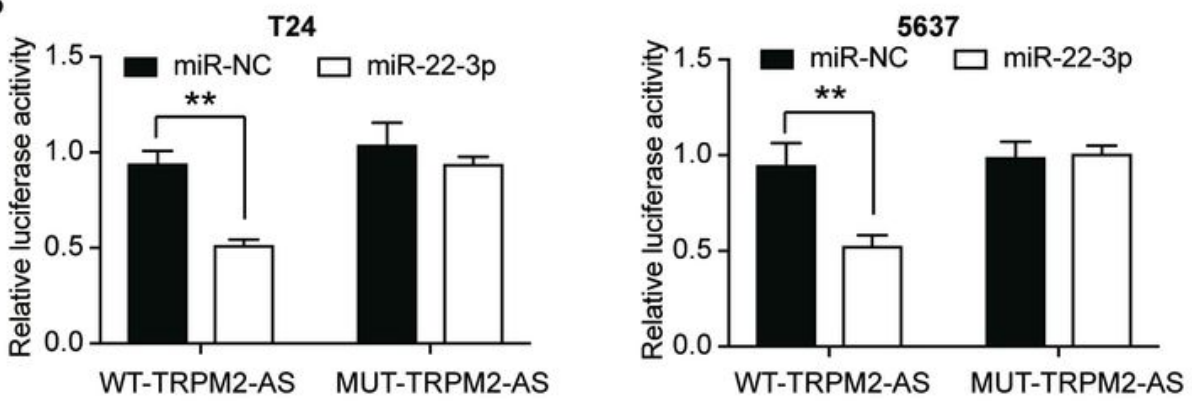

C

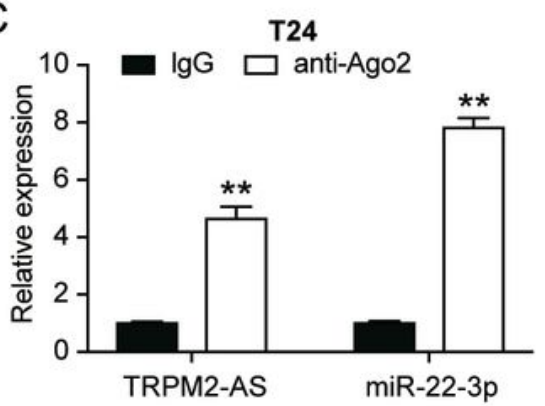

$\mathrm{D}$

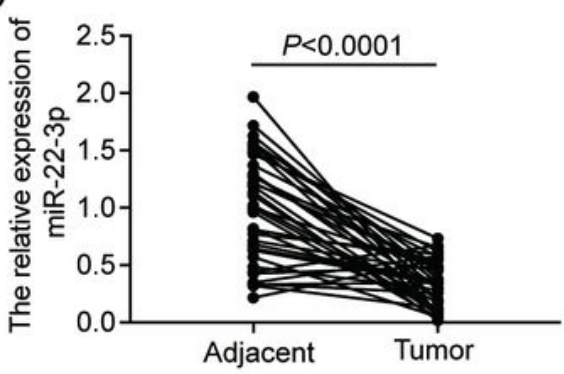

$\mathrm{F}$
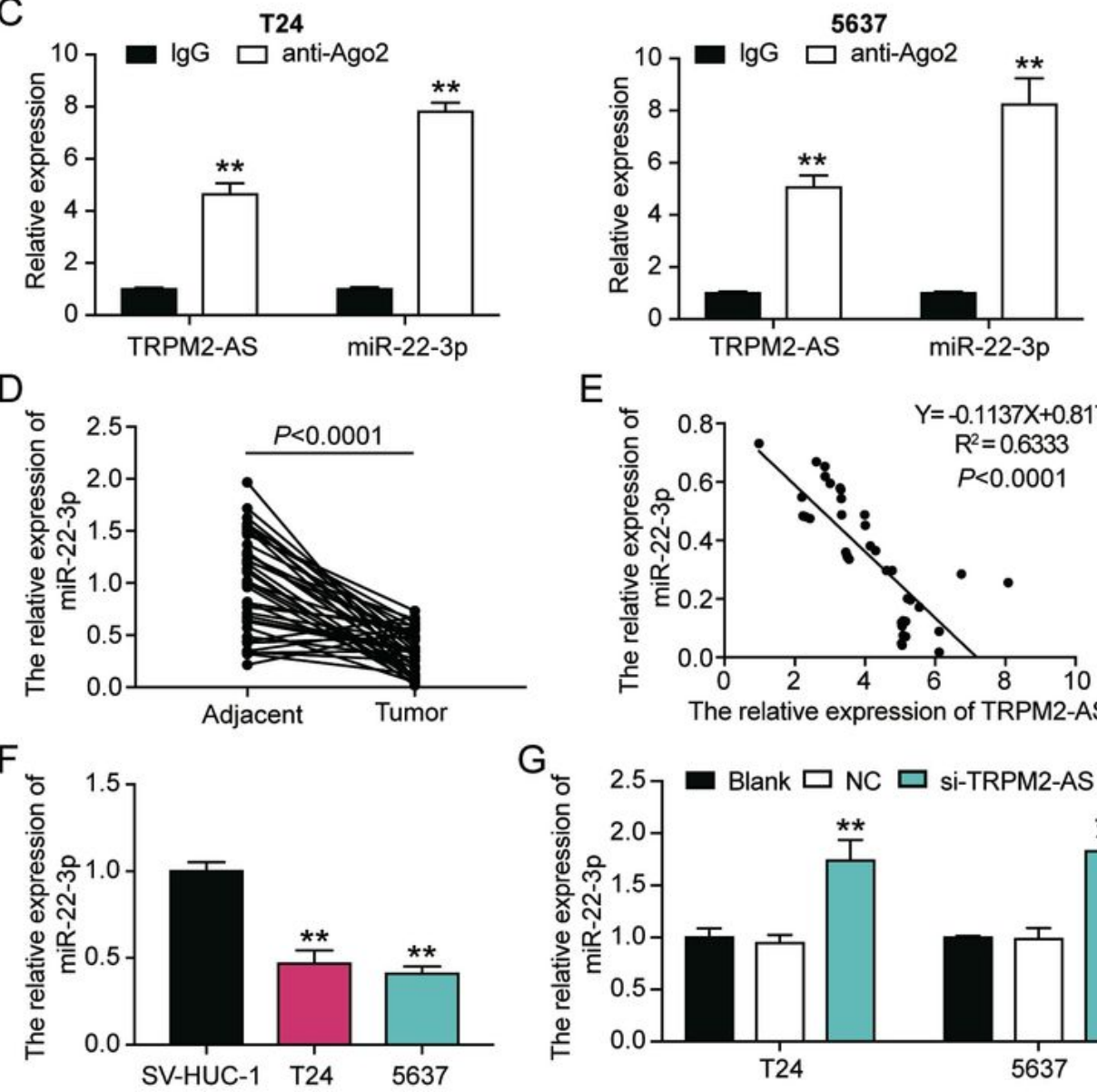

$E$

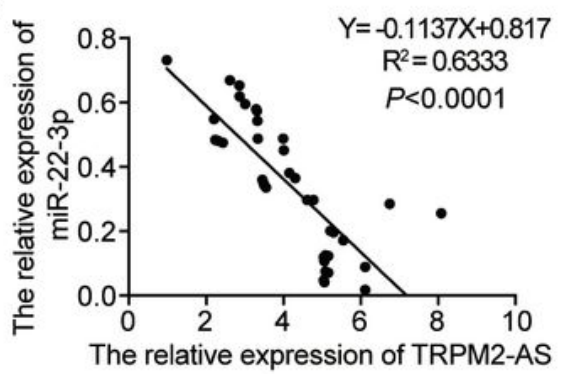

$G$

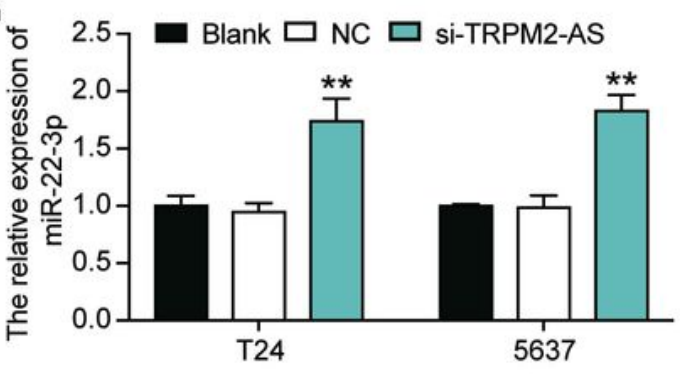

Figure 3

The negative correlation between MiR-22-3p and TRPM2-AS. A. The combine region sequences of TRPM2-AS and miR-22-3p. B. Luciferase reporter assay was used to assess the targeting relationship between TRPM2-AS and miR-22-3p. ${ }^{* * P}<0.001$, ANOVA. C. RIP assay was used to further confirm the targeting relationship between TRPM2-AS and miR-22-3p. **P $<0.001$ compared with IgG group, ANOVA. D. the expression of miR-22-3p was down-regulated in BLCA tissues compared with normal adjacent 
tissues. $\mathrm{N}=38$, Student's t-test. E. TRPM2-AS expression had a negative relationship with miR-22-3p expression. F. The expression of miR-22-3p was reduced in T24 and 5637 cells compared with SV-HUC-1 cells. ${ }^{* *} \mathrm{P}<0.001$ compared with SV-HUC-1 cells, ANOVA. G. The expression of miR-22-3p was increased after transfecting si-TRPM2-AS in T24 and 5637 cells by qRT-PCR analysis. ${ }^{*} \mathrm{P}<0.001$ compared with blank group, ANOVA.
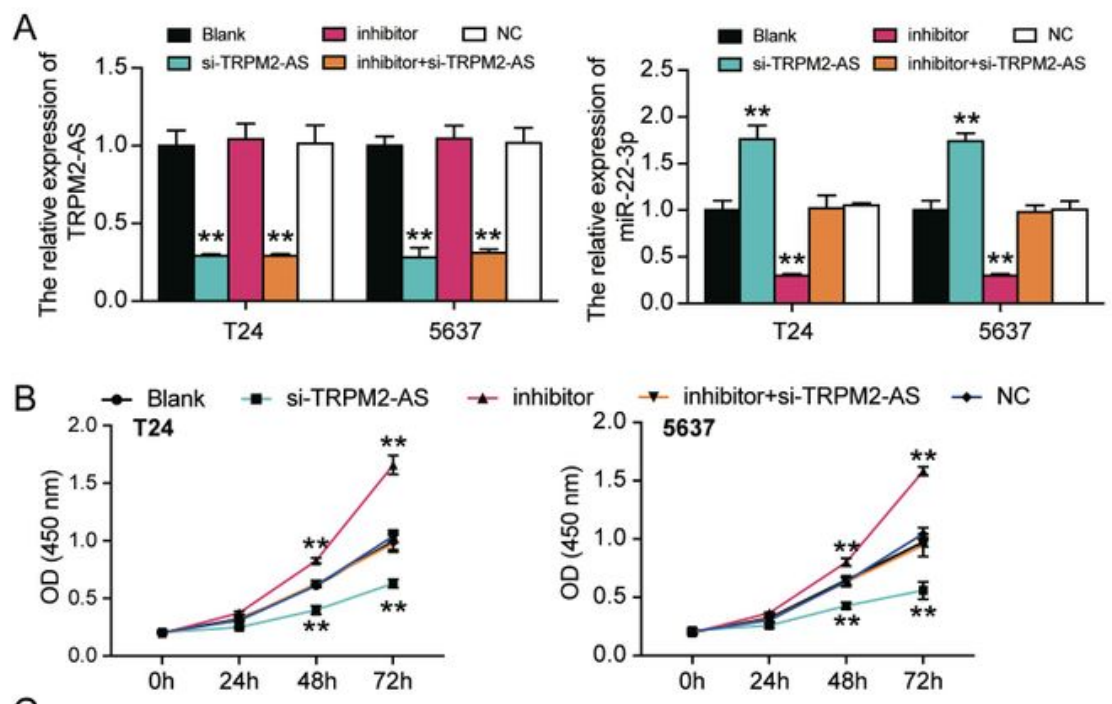

C
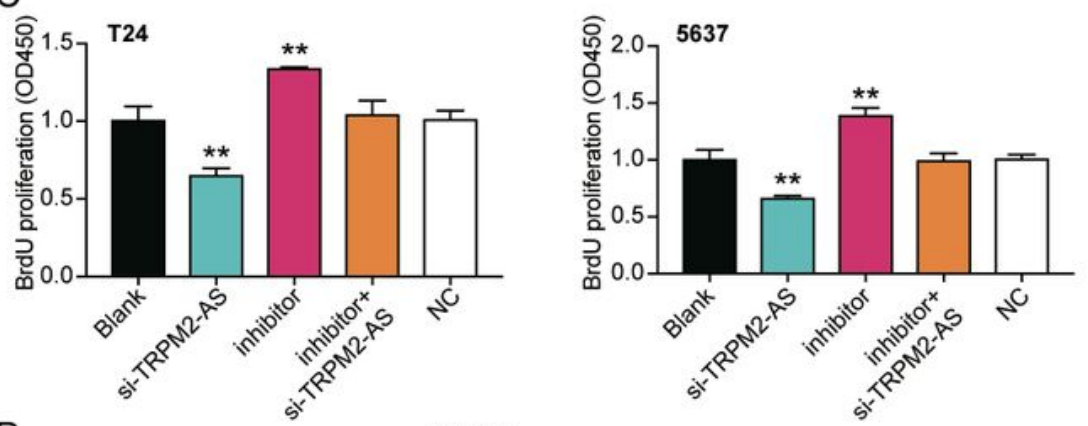

D
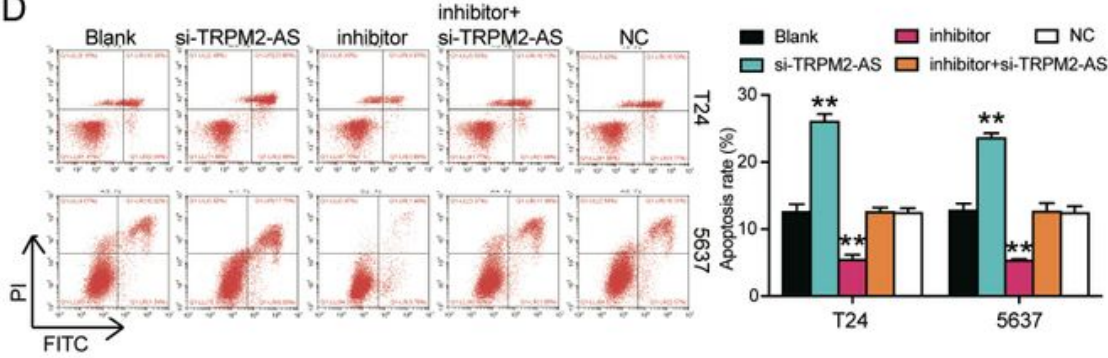

E
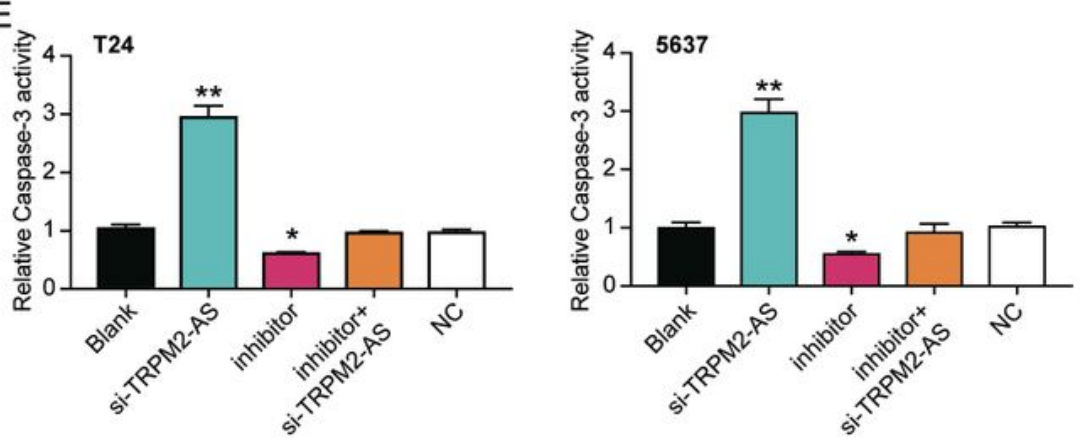

Figure 4 
MiR-22-3p inhibitor promoted cell viability and cell proliferation, but inhibited cell apoptosis in BLCA cells which was regulated by TRPM2-AS. A. The transfection efficiency of miR-22-3p inhibitor and TRPM2-AS siRNA. B. CCK-8 assay was used to observe the cell viability in T24 and 5637 cells after transfecting miR22-3p inhibitor, si-TRPM2-AS, negative control, blank control and co-transfected miR-22-3p inhibitor and si-TRPM2-AS. C. BrdU assay was used to observe the cell proliferation in T24 and 5637 cells after transfecting miR-22-3p inhibitor, si-TRPM2-AS, negative control, blank control and co-transfected miR-22$3 p$ inhibitor and si-TRPM2-AS. D. Flow cytometry was employed to measure the cell apoptosis in T24 and 5637 cells after transfecting miR-22-3p inhibitor, si-TRPM2-AS, negative control, blank control and cotransfected miR-22-3p inhibitor and si-TRPM2-AS. E. Caspase-3 activation assay was employed to measure the caspase-3 activation in BLCA cells after transfecting miR-22-3p inhibitor, si-TRPM2-AS, negative control, blank control and co-transfected miR-22-3p inhibitor and si-TRPM2-AS. ${ }^{*} \mathrm{P}<0.05$, ${ }^{\star *} \mathrm{P}<$ 0.001 compared with blank group, ANOVA. 
Wild-type GINS2 3'UTR 5 ' gccugagccaguggaGGCAGCUc 3'

hsa-miR-22-3p 3' ugucaagaaguugaCCGUCGAa 5'

Mutant GINS2 3'UTR 5' gccugagccaguggaAAUCCGAc 3'

B

T24

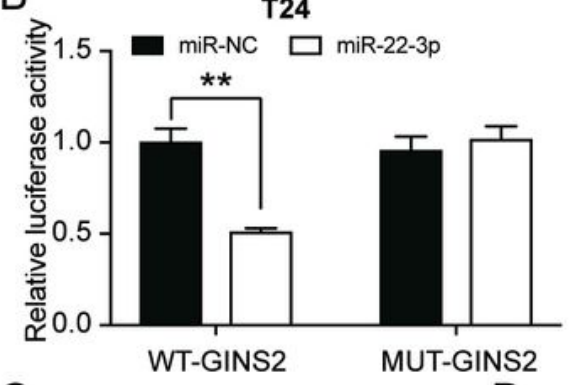

C

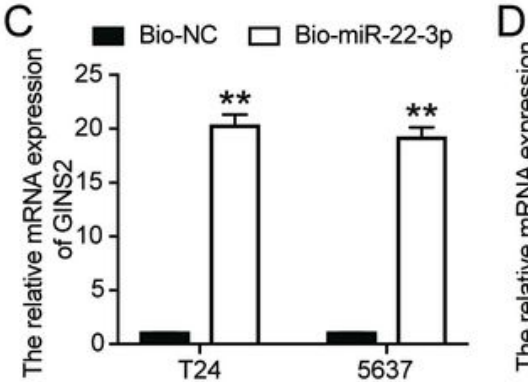

$\mathrm{F}_{\mathrm{c}}$

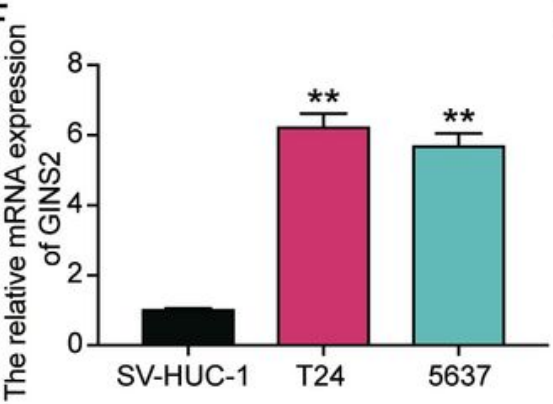

$\mathrm{H}$

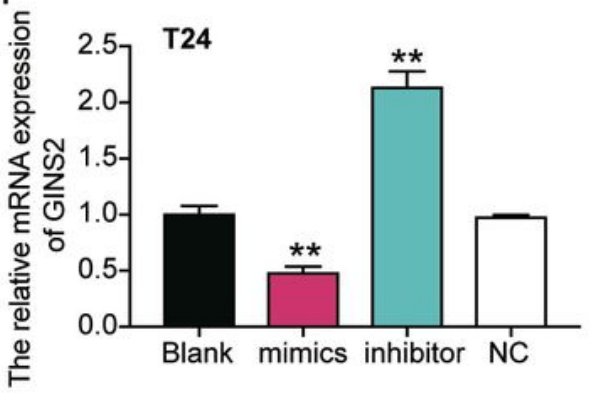

5637
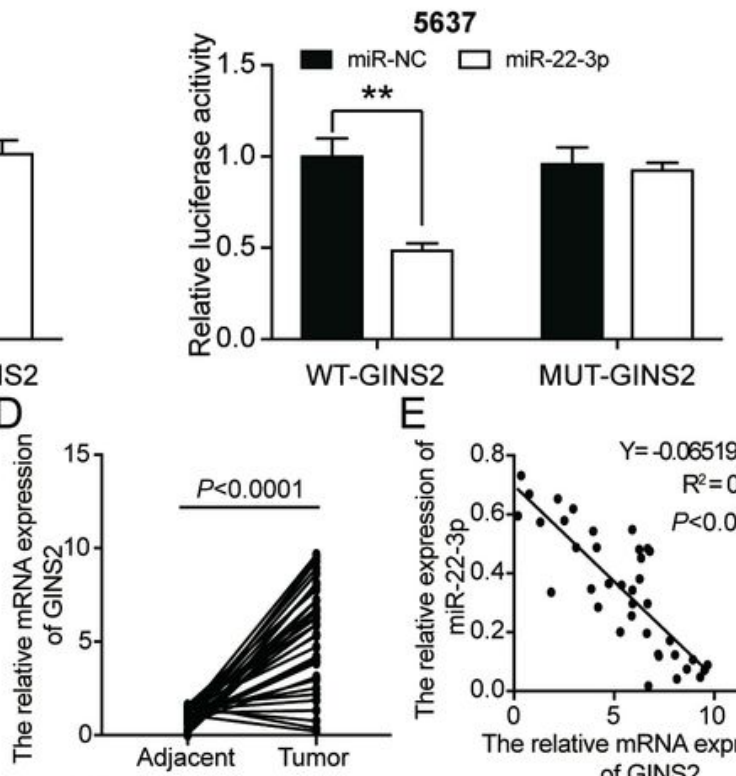

$\mathrm{G}$
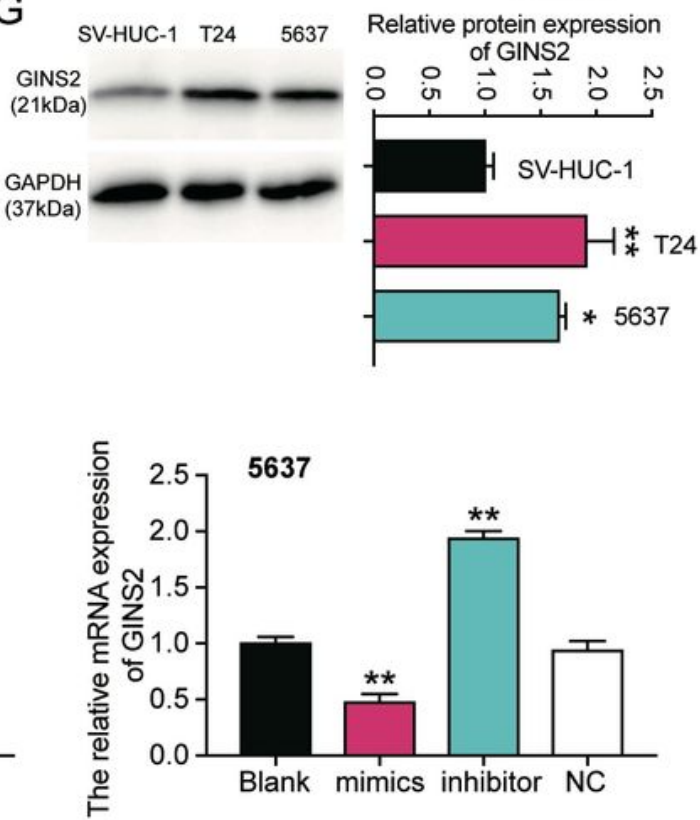

\section{Figure 5}

GINS2 was the downstream target gene of miR-22-3p. A. The binding site of GINS2 3'UTR for miR-22-3p.

B. Luciferase reporter assay was used to measure the targeting relationship between miR-22-3p and GINS2. ${ }^{*} \mathrm{P}<0.001$, ANOVA. C. RNA pull-down assay was used to further confirm the targeting relationship between TRPM2-AS, miR-22-3p and GINS2. ${ }^{*} \mathrm{P}<0.001$ compared with bio-NC group, ANOVA. D. The GINS2 mRNA expression was more in BLCA tissues than that in adjacent normal tissues. $N=38$, 
Student's t-test. E. MiR-22-3p expression had a negative relationship with GINS2 expression. F. GINS2 mRNA expression was higher in T24 and 5637 cell lines than that in SV-HUC-1 cells. ${ }^{\star *} \mathrm{P}<0.001$ compared with SV-HUC-1 cells, ANOVA. G. GINS2 protein expression was higher in T24 and 5637 cell lines than that in SV-HUC-1 cells. ${ }^{*} \mathrm{P}<0.05,{ }^{*} \mathrm{P}<0.001$ compared with SV-HUC-1 cells, ANOVA. H. GINS2 mRNA expression was increased after transfecting miR-22-3p inhibitor, while GINS2 mRNA expression was reduced after transfecting miR-22-3p mimic. ${ }^{*} \mathrm{P}<0.001$ compared with blank group, ANOVA.
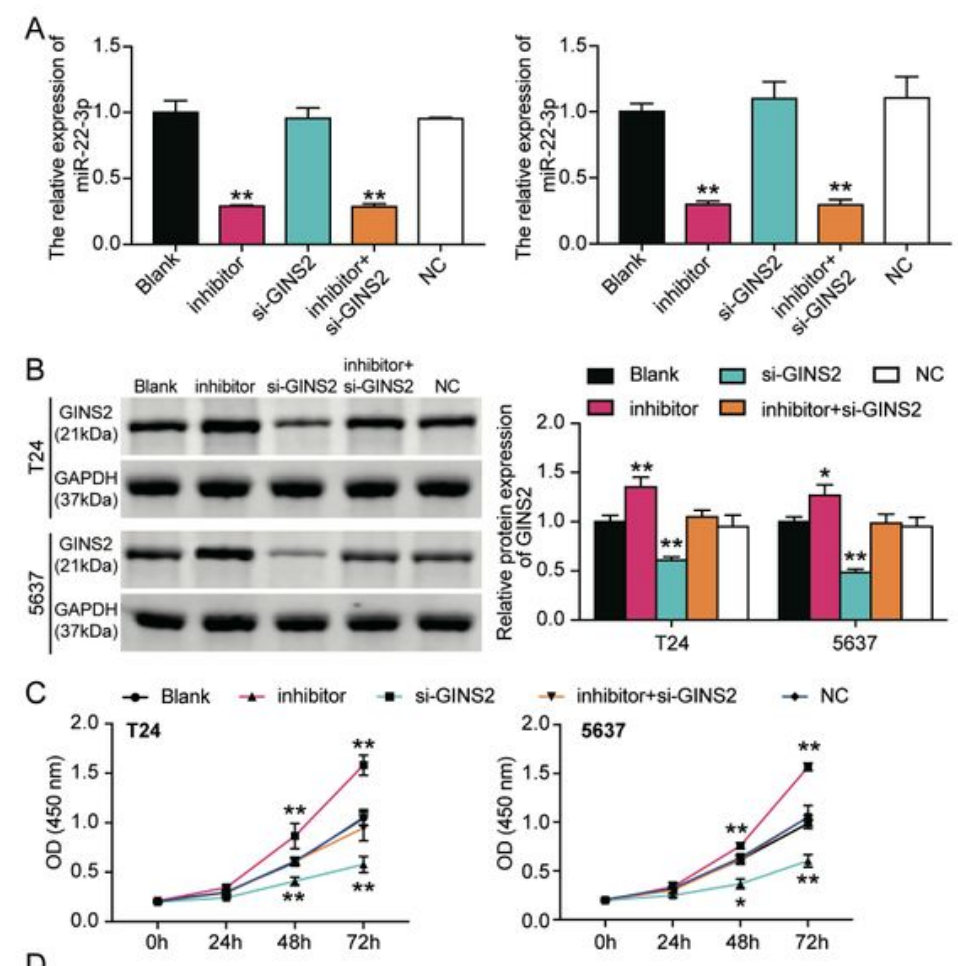

D
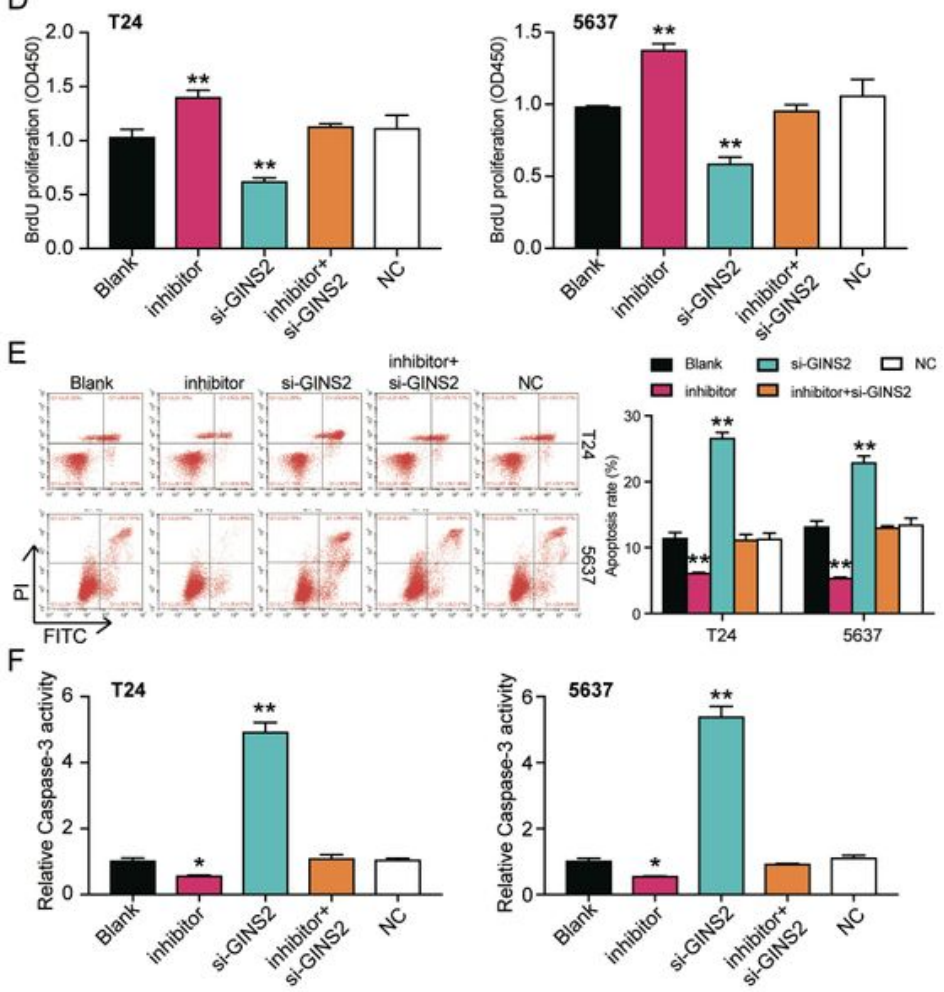

Figure 6 
GINS2 silence inhibited cell viability and cell proliferation while promoted cell apoptosis in bladder cancer cells which was regulated by miR-22-3p. A. The transfection efficiency of miR-22-3p inhibitor and GINS2 siRNA in the two cell lines. B. The expression of GINS2 protein was detected by western blot in BLCA cells after transfecting with miR-22-3p inhibitor, si-GINS2, negative control, blank control and co-transfected miR-22-3p inhibitor and si-GINS2. C. CCK-8 assay was used to observe the cell viability in T24 and 5637 cell lines after transfecting miR-22-3p inhibitor, si-GINS2, negative control, blank control and cotransfected miR-22-3p inhibitor and si-GINS2. D. BrdU assay was used to observe the cell proliferation in T24 and 5637 cell lines after transfecting miR-22-3p inhibitor, si-GINS2, negative control, blank control and co-transfected miR-22-3p inhibitor and si-GINS2. E. Flow cytometry was employed to measure the cell apoptosis after transfecting miR-22-3p inhibitor, si-GINS2, negative control, blank control and cotransfected miR-22-3p inhibitor and si-GINS2. F. Caspase-3 activity assay was employed to measure the caspase-3 activity after transfecting miR-22-3p inhibitor, si-GINS2, normal control, blank control and cotransfected miR-22-3p inhibitor and si-GINS2. ${ }^{*} \mathrm{P}<0.05,{ }^{\star} \mathrm{P}<0.001$ compared with blank group, ANOVA. NC: negative control. 